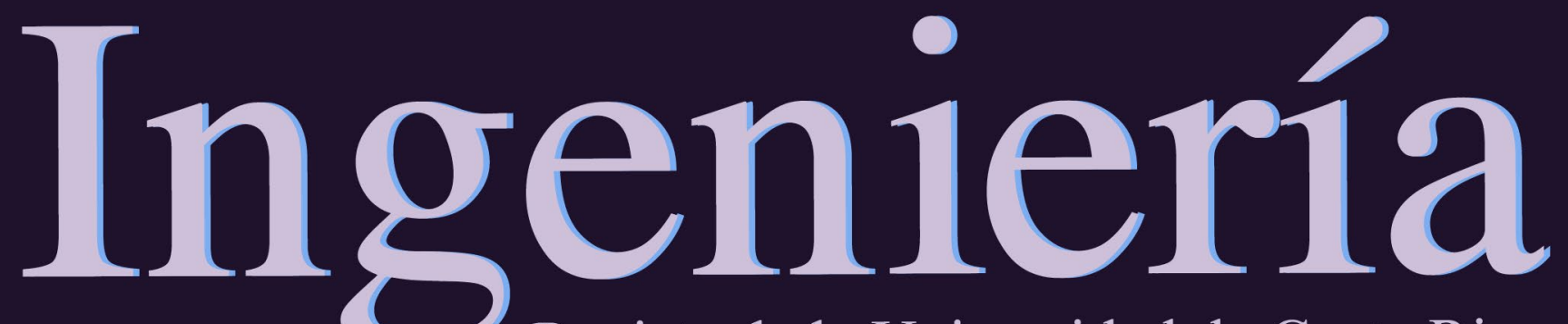

Revista de la Universidad de Costa Rica

ENERO / JUNIO 2020 - VOLUMEN 30 (1)
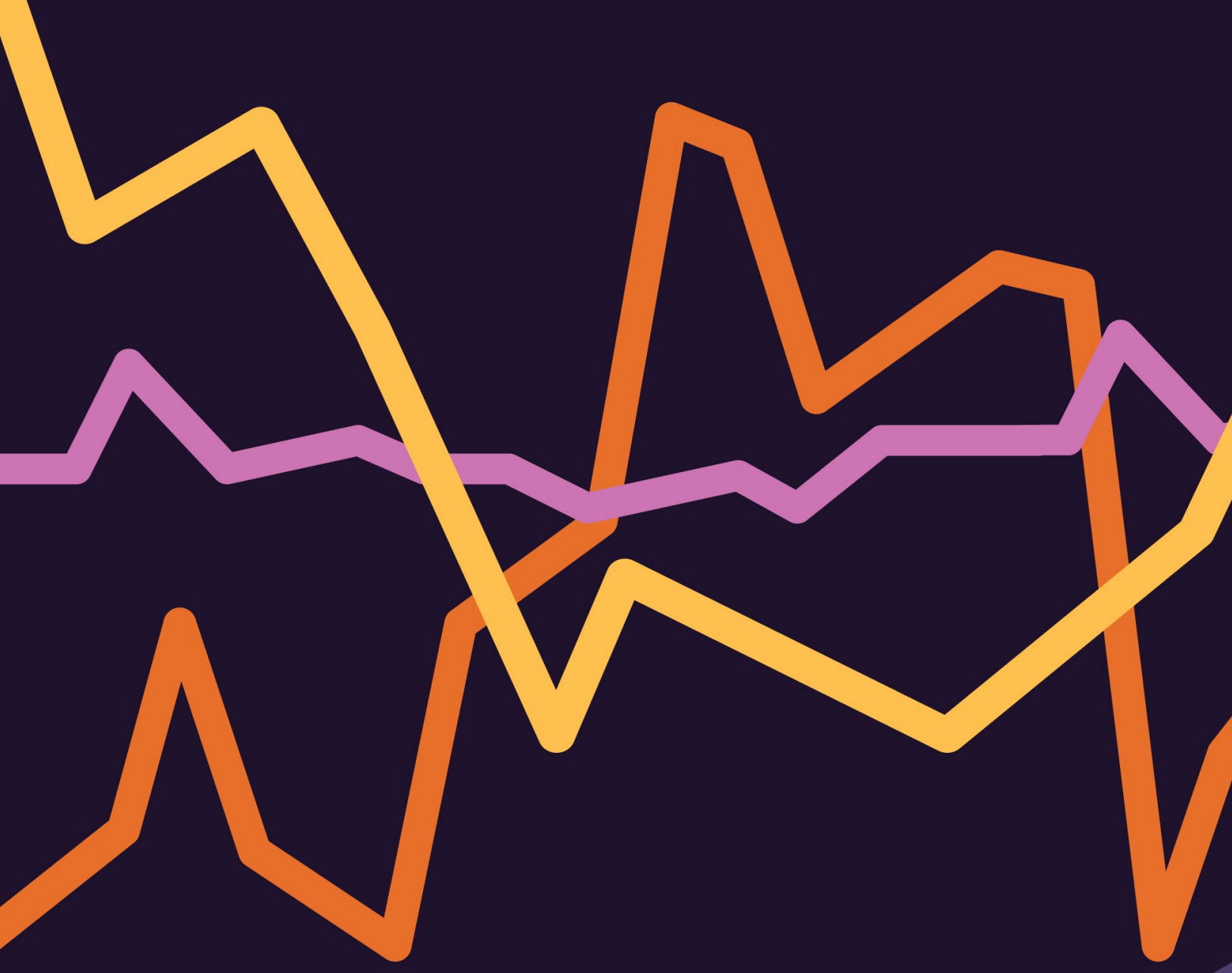


\title{
Evaluación del desempeño de un catalizador de níquel/alúmina en distintas condiciones de operación para la producción de hidrógeno mediante reformado de metanol en fase acuosa (APR)
}

\author{
Evaluation of the Performance of a Nickel/Alumina Catalyst Under \\ Different Operating Conditions to Produce Hydrogen by Aqueous \\ Phase Methanol Reforming (APR)
}

\author{
Ing. Felipe Cartín Muñoz, \\ Ingeniero Químico, Enginner I en Microvi Biotech Inc., \\ California, Estados Unidos \\ phcartin@gmail.com \\ Ing. Adolfo Ulate Brenes, Mag. \\ Profesor e Investigador de la Escuela de Ingeniería Química, \\ Universidad de Costa Rica, San José, Costa Rica \\ adolfo.ulate@ucr.ac.cr
}

Recibido: 7 de setiembre 2019

Aceptado: 29 de noviembre 2019

\section{Resumen}

El objetivo del proyecto de investigación fue determinar el efecto de la temperatura, concentración de metanol y cantidad de catalizador en la tasa de producción de hidrógeno por medio de un proceso de reformado en fase acuosa, utilizando un catalizador de níquel depositado en alúmina comercial.

El catalizador se sintetizó utilizando el método de impregnación incipiente con nitrato de níquel, se calcinó y se redujo utilizando una corriente al $5 \%$ de hidrógeno, con un flujo de 250x10-3 $1 \mathrm{~min}^{-1}$, a $723.15 \mathrm{~K}$ $\left(450{ }^{\circ} \mathrm{C}\right)$ por un periodo de $4 \mathrm{~h}$. El catalizador sintetizado se caracterizó y se determinó el área superficial, cristalinidad, composición, así como se realizaron micrografías para comprobar su morfología.

Para el estudio del efecto de las variables en la tasa de producción de hidrógeno se realizó un diseño experimental Taguchi L8, con tres variables y dos niveles: temperatura $473.15 \mathrm{~K}$ y $498.15 \mathrm{~K}$, concentración de metanol $2 \%$ v/v y $6 \%$ v/v; y cantidad de catalizador $2,5 \% \mathrm{~m} / \mathrm{m}$ y $5 \% \mathrm{~m} / \mathrm{m}$.

Se encontró que el catalizador utilizado presenta un área de $88 \mathrm{~m}^{2} / \mathrm{g}$, con poca cristalinidad, considerándose amorfo, con un porcentaje de níquel cercano al 3\%. Con respecto al diseño estadístico se comprobó que solo la temperatura es significativa en la taza de producción de hidrógeno y que es mejor trabajar a $498.15 \mathrm{~K}$.

Palabras clave:

APR, Metanol, Reformado en fase acuosa, Producción de hidrógeno, Catalizador niquel-alúmina 


\begin{abstract}
The main objective of this study were to determinate the effect of the temperature, methanol concentration and catalyst ratio, in the rate of hydrogen production, using aqueous phase reforming reaction. The catalyst used was impregnated nickel in commercial alumina.

The catalyst was produced by the incipient nickel nitrate impregnation method. Furthermore, was calcined and reduced, using hydrogen (5\%), with a flow of $250 \times 10^{-3} \mathrm{l} \mathrm{min}^{-1}$, at $723.15 \mathrm{~K}\left(450{ }^{\circ} \mathrm{C}\right)$ for $4 \mathrm{~h}$. The synthesized catalyst was characterized, and the surface area, crystallinity and composition were determined, as well as micrographs were taken to verify its morphology.

To find the effect of the variables in the rate of hydrogen production, a Taguchi L8 statistical design was used with three variables and two levels: temperature $473.15 \mathrm{~K}$ y $498.15 \mathrm{~K}$, methanol concentration $2 \% \mathrm{v} / \mathrm{v}$ y $6 \% \mathrm{v} / \mathrm{v}$; and catalyst fraction used $2,5 \% \mathrm{~m} / \mathrm{m}$ y $5 \% \mathrm{~m} / \mathrm{m}$.

One of the findings was that the catalyst used has an area of $88 \mathrm{~m}^{2} / \mathrm{g}$, with little crystallinity therefore, it was considered amorphous, with a nickel content of approximately of $3 \%$. According to the statistical design, it was observed that only the temperature is significant in the rate of hydrogen production and the optimal temperature that favors hydrogen production is $498.15 \mathrm{~K}$.
\end{abstract}

\title{
Keywords:
}

APR, Aqueous phase reforming, Methanol, Nickel catalysts; Alumina; $\mathrm{H}_{2}$ production 


\section{INTRODUCCIÓN}

Los combustibles fósiles y sus derivados como fuente energética, así como su alta demanda a nivel mundial han sido de suma importancia en el rol del desarrollo de la sociedad moderna, lo que los ha vuelto commodities que se consumen de manera masiva. Las fluctuaciones de los precios del petróleo y de sus derivados, su influencia en la contaminación ambiental y las proyecciones de desabastecimiento en un futuro cercano hacen que se busquen alternativas a estos productos que sean menos nocivas para el ambiente y que permitan suplir la matriz energética de manera diversificada. Se han realizado distintos esfuerzos en esta área: uso de energías renovables, biocombustibles de distintas fuentes (semillas, plantas, algas); que en muchos casos compiten con biomasa con fines alimenticios. Integrando conceptos de sostenibilidad, aprovechamiento de los recursos y subproductos, se han buscado otras fuentes de biomasa para la producción de productos de valor agregado, de ahí nace el concepto de la biorrefinería, la cual aprovecha materias primas biomásicas y en la mayoría de los casos, utilizando los residuos de plantas, material lignoceluloso, y subproductos de procesos industriales ya establecidos (Jose y Shibu, 2015).

De las opciones antes mencionadas en el área de los biocombustibles surge la alternativa de producir hidrógeno por medio de reformado de biomasa en fase acuosa APR (Aqueous Phase Reforming). El proceso puede generar hidrógeno, gas de síntesis, alcanos, y grupos monofuncionales a bajas presiones en ambientes acuosos y temperaturas de alrededor de $500 \mathrm{~K}$ (Cammack, Frey y Robson, 2001).

Otra ventaja de la producción de hidrógeno integrado a su producción a través de fuentes renovables es la posibilidad del uso del hidrógeno en celdas de combustible, lo que genera energía eléctrica a bajas temperaturas y cuyo único subproducto es el agua (Shabaker, Davda, Huber, Cortight y Dumesic, 2003).

\section{ASPECTOS GENERALES}

\subsection{Reformado en fase acuosa (APR)}

La utilización de compuestos orgánicos oxigenados en procesos de transformación térmicos ha sido empleada con frecuencia por medio de catálisis heterogénea; por ejemplo, el reformado con vapor SR; sin embargo, presenta grandes desventajas energéticas y se ha tratado recientemente de buscar sistemas con menores requerimientos energéticos y manejo de fases de reacción líquidas y no gaseosas. Los procesos de reformado en fase líquida APR utilizan presiones superiores a la presión de vapor de la mezcla de reacción a la temperatura de reacción, por lo que esta siempre ocurre en fase líquida.

La temperatura y presión de un sistema APR favorece la termodinámica de la reacción de desplazamiento de vapor-gas (WGS, water-gas shift reaction) aumentando la conversión de monóxido de carbono en un único reactor, asegurando la producción de hidrógeno en condiciones casi puras junto con dióxido de carbono. Debido a las presiones utilizadas en estos procesos que varían entre 
1.5 MPa y 5.1 MPa los métodos convencionales de separación criogénica y membranas se pueden aplicar para separar el hidrógeno del dióxido de carbono. El reformado de vapor SR utiliza presiones bajas; por ende, se requiere presurización posterior para la etapa de separación. Al trabajar en esos ámbitos de presión y temperatura el monóxido de carbono se transforma en dióxido de carbono al consumir agua del sistema, liberando más hidrógeno durante el proceso, es una reacción en serie y necesaria para la generación de hidrógeno (Shan, 2014).

Bajo condiciones de operación específicas en los sistemas de reformado en fase acuosa y con la utilización de un catalizador adecuado, se favorecen desde un punto de vista termodinámico las reacciones de WGS, la metanación y la síntesis de Fischer-Thropsch (Shabaker et al., 2003):

$$
\begin{aligned}
\mathrm{C}_{\mathrm{n}} \mathrm{H}_{2 \mathrm{n}+2} \mathrm{O}_{n}+\mathrm{H}_{2} \mathrm{O} & \rightleftharpoons \mathrm{nCO}+(2 \mathrm{n}+1) \mathrm{H}_{2} \quad \Delta H>0 \\
\mathrm{CO}+\mathrm{H}_{2} \mathrm{O} & \rightleftharpoons \mathrm{CO}_{2}+\mathrm{H}_{2} \quad \Delta H<0 \\
\mathrm{CO}+3 \mathrm{H}_{2} & \rightleftharpoons \mathrm{CH}_{4}+\mathrm{H}_{2} \mathrm{O} \Delta H<0 \\
\mathrm{CO}_{2}+4 \mathrm{H}_{2} & \rightleftharpoons \mathrm{CH}_{4}+2 \mathrm{H}_{2} \mathrm{O} \Delta H<0 \\
(2 \mathrm{n}+1) \mathrm{H}_{2}+\mathrm{nCO} & \rightarrow \mathrm{C}_{\mathrm{n}} \mathrm{H}_{2 \mathrm{n}+2}+n \mathrm{H}_{2} \mathrm{O} \Delta H<0 \\
(2 \mathrm{n}) \mathrm{H}_{2}+\mathrm{nCO} & \rightarrow \mathrm{C}_{\mathrm{n}} \mathrm{H}_{2 \mathrm{n}}+n \mathrm{H}_{2} \mathrm{O} \quad \Delta H<0
\end{aligned}
$$

Por lo anterior, la producción de hidrógeno no solo se define desde el punto de vista termodinámico, ya que el equilibrio de la reacción (4), es del orden de 1010 a $500 \mathrm{~K}$, por lo que favorece el consumo del hidrógeno producido a las temperaturas de operación. (Davda, Shabaker, Huber, Cortringht y Dumestic, 2005). Otras consideraciones con respecto a la temperatura, es que se fija en función de la presión de vapor de la materia prima, la coquificación del catalizador, o la descomposición térmica de los reactivos.

La función del catalizador en las reacciones APR se relacionan con la ruptura selectiva de los enlaces carbono carbono (C-C), junto con la limitación de la ruptura de los enlaces carbono oxígeno (C-O) de la biomasa, lo que favorece la formación de hidrógeno en lugar de alcanos de cadenas cortas (Coronado et al., 2016). La ruptura selectiva de los enlaces C-C se logra utilizando catalizadores depositados con metales del grupo VI como platino, níquel, rutenio, paladio; además estos facilitan la reacción de desplazamiento agua-vapor (Cruz, Ribeiro, Aranda y Souza, 2008). Otras consideraciones sobre este tipo de catalizadores son los costos asociados al metal activo, y aunque el platino muestra altas selectividades y rendimientos el costo de este hace que se exploren otros metales como el níquel, que presenta alta actividad catalítica comparada con el platino, pero muy baja selectividad (Coronado et al., 2016). Otros problemas asociados al uso de níquel como catalizador es que presenta alto decaimiento de actividad debido al cambio en el estado de oxidación, sinterización y cambio en la estructura. (Stekrova, Rinta-Paavola y Karinen, 2018) 


\section{EQUIPO, MATERIALES Y METODOLOGÍA EXPERIMENTAL}

\subsection{Equipos principales}

- Reactor de presión HP/HT 4570, Parr Instument Company

- $\quad$ Cromatógrafo de gases con detector de conductividad térmica (TCD), modelo HP 6890, marca Hewlett Packard

- $\quad$ Balanza analítica Adam AFP-360L

- Capsula de reducción, capacidad de $35 \mathrm{~g}$

\subsection{Metodología Experimental}

\subsubsection{Elaboración del catalizador}

Para lograr un catalizador homogéneo se utilizaron de $25 \mathrm{~g}$ de alúmina (SIGMA-ALDRICH) a los que se le agregaron $3.83 \mathrm{~g}$ de nitrato de níquel hexahidratado (SIGMA-ALDRICH, $\geq$ 97.0). La sal de níquel se disolvió en la menor cantidad de agua posible para luego ser transferida por medio de un gotero a la alúmina colocada en un mortero. Se homogenizó la mezcla hasta obtener un color verde y uniforme en toda la masa. Una vez homogéneo, el catalizador se seca por un periodo de 12 horas a $105^{\circ} \mathrm{C}$.

Después del proceso de secado, el catalizador se sometió a un proceso de reducción en el cual se hizo pasar una corriente de 5\% de hidrógeno, 95\% argón (TRIGAS) por el catalizador, en una capsula diseñada para tal fin. Las condiciones del gas utilizado fueron: presión ambiente, temperatura de $450{ }^{\circ} \mathrm{C}$ (Munnik, de Jongh, y de Jong, 2015) y (Rase, 2016), flujo de $250 \mathrm{ml} \mathrm{min}^{-1}$, durante 4 horas. El catalizador reducido se almacenó en la misma cápsula de reducción pasando una corriente de nitrógeno puro para evitar oxidación del níquel con el oxígeno del aire.

El proceso de producción de catalizador se realizó varias veces de acuerdo con las necesidades del diseño experimental del proceso de reformado en fase acuosa.

\subsubsection{Caracterización del catalizador}

Área

La medición se realizó en el Laboratorio de Fisicoquímica de la Escuela de Química de la Universidad de Costa Rica. Se aplicó la metodología de curva de absorción (BET). Se llevó la muestra, se pesó $0.5 \mathrm{~g}$ y se desgasificó con una corriente de helio. Por medio de la adsorción de nitrógeno se relacionó el área superficial. 


\section{Composición}

Se le realizó una prueba de composición por medio de absorción atómica para la cual se requirió preparar una muestra de $35 \mathrm{~g}$. El análisis se realizó en el Laboratorio de Servicios Analíticos (LASA) en la Escuela de Química en la Universidad de Costa Rica.

\section{Cristalinidad}

Análisis por difractometría de rayos-X (XRD) empleando el equipo Panalytical modelo Empyream usando silicio como patrón de verificación. El análisis se levó a cabo en un tubo de cobre con una potencia de $45 \mathrm{kV}$ y una corriente de $40 \mathrm{~mA}$ usando un barrido de $5^{\circ}$ a los $100^{\circ}$. El análisis se realizó en el Centro de Investigación y Extensión en Materiales CIEMTEC de la Escuela de Ciencia e Ingeniería de los Materiales en el Instituto Tecnológico de Costa Rica.

\section{Microscopía}

Se hizo un análisis de microscopía SEM con un acople EDX. El ensayo se realizó en el Centro de Investigación en Estructuras Microscópicas (CIEMIC) en la Universidad de Costa Rica utilizando un microscopio electrónico de barrido (SEM), modelo S-3700N número de serie 371238-03 con un voltaje de $15000 \mathrm{~V}$ y un aumento de 42. La muestra se recubrió con una capa de oro para mejorar la resolución. Se usó un EDX de iridio.

\section{Reacción}

Se acondicionó el reactor a presión HP/HT 4570 para realizar los experimentos. Se cargó el reactor con la disolución de metanol (Laboratorios Quimar, anhidro 99,9\%) en agua y se agregó el catalizador reducido de acuerdo con las condiciones que se muestran más adelante. Se purgó el aire dentro del reactor pasando una corriente de nitrógeno por un período de $5 \mathrm{~min}$. Finalizada la purga, el reactor se presurizó con nitrógeno a una presión de $344.64 \mathrm{kPa}$ (50 psi) y $448.04 \mathrm{kPa}$ (65 psi) para las temperaturas de $473.15 \mathrm{~K}$ y $498.15 \mathrm{~K}$ respectivamente. Se encendió el sistema de calentamiento del reactor y se dejó llegar a la temperatura y presión correspondiente al diseño estudiado. Al alcanzar la temperatura de reacción se tomó como tiempo cero de la reacción y se dejó correr la misma por un período de dos horas, momento en el que se tomó la muestra gaseosa del reactor. Para la extracción de la muestra se abrió la válvula de muestreo del equipo, se purgó el gas de la línea de muestreo por unos segundos manteniendo la presión del reactor. La muestra se tomó con una jeringa con volumen de $10 \mathrm{ml}$, y se inyectó en un vial de $5 \mathrm{ml}$. Seguidamente, se redujo la presión del vial perforando el septum en la tapa con una aguja de jeringa bajo el agua hasta nivelar con presión ambiente.

Las condiciones de reacción se definieron por medio de la revisión bibliográfica de acuerdo con criterios establecidos por estudios previos dados por Tungal y Shende (2013), Miranda Morales (2017)y Shabaker et al. (2003). Para el estudio de los factores seleccionados y los niveles de estudio, se realizó un diseño Taguchi L8 $\left(2^{3}\right)$, en el cual se realizan ocho corridas experimentales con tres variables y dos niveles como se muestra en la Tabla 1. 
Tabla 1. Condiciones de niveles de las variables a analizar en el diseño estadístico Taguchi L8

\begin{tabular}{cccc}
\hline Corrida & Temperatura, $T /(K)$ & $\begin{array}{c}\text { Concentración de } \\
\text { Metanol, } C n /(\% \mathrm{v} / \mathrm{v})\end{array}$ & $\begin{array}{c}\text { Cantidad Catalizador, } \\
C C /(\% \mathrm{~m} / \mathrm{m})\end{array}$ \\
\hline 1 & 473.15 & 2 & 2.5 \\
2 & 473.15 & 2 & 5 \\
3 & 473.15 & 6 & 2.5 \\
4 & 473.15 & 6 & 5 \\
5 & 498.15 & 2 & 2.5 \\
6 & 498.15 & 2 & 5 \\
7 & 498.15 & 6 & 2.5 \\
8 & 498.15 & 6 & 5 \\
\hline
\end{tabular}

Medición de hidrógeno

Para evaluar el desempeño del catalizador bajo las condiciones de cada corrida experimental se utilizará la cantidad de hidrógeno producido, definida como $\mu \mathrm{mol}_{\mathrm{H} 2} \mathrm{~s}^{-1} \mathrm{~g}_{\mathrm{cat}}{ }^{-1}$.

La muestra del vial de $5 \mathrm{ml}$ que se extrajo del reactor se inyectó en el cromatógrafo de gases (GC) siguiendo el procedimiento definido por Incer (2016).

Se construyó una curva de calibración con hidrógeno ultra puro, realizando mezclas que van desde hidrógeno puro hasta un $5 \%$ en volumen (7 puntos). Para esto se tomó un bulbo de vidrio con septum incorporado y se purgó varias veces con hidrógeno ultra puro hasta obtener un volumen apreciable para hacer la curva. Se tomaron volúmenes con la jeringa de $250 \mu$ y se mezclaron con aire atmosférico, seguidamente, se inyectaron en el GC.

Respecto a las condiciones de operación del GC se tiene una temperatura de inyección de $378.15 \mathrm{~K}$, se utilizó un detector de conductividad térmica (TCD) para diferenciar al hidrógeno usando nitrógeno puro como gas con un flujo de $4 \mathrm{ml} / \mathrm{min}$ de arrastre que operó a $503.15 \mathrm{~K}$. Se utilizó una columna de sílice fundida capilar (Carboxen 1010 Plot $30 \mathrm{~m} \mathrm{x} 0.53 \mathrm{~mm}$ ), a una temperatura de $323.15 \mathrm{~K}$. Antes de pasar la muestra, se realizaron muestreos con aire atmosférico y se analizaron en el GC para distinguir trazas remantes de oxígeno en las muestras al utilizar aire ambiente como gas de mezcla. 


\section{ANÁLISIS DE RESULTADOS}

\subsection{Catalizador}

El área superficial de la alúmina comercial que se utilizó como soporte para el agente metálico en las reacciones de reformado reportada por el proveedor del compuesto, tiene un promedio de malla de $0.104 \mathrm{~mm}$ (mesh 150), y con un intervalo de área de $85 \mathrm{~m}^{2} / \mathrm{g}$ a $115 \mathrm{~m}^{2} / \mathrm{g}$. En general los soportes de catalizadores para reacciones heterogéneas se consideran aptos a valores cercanos a los 100 m²/g (Chang, Kuo, Tsay y Hsieh, 2003). Después de realizar la curva de absorción (BET) para determinar el área del soporte, se logró verificar que tiene un área superficial de $88 \mathrm{~m}^{2} / \mathrm{g}$ lo cual está dentro del intervalo que indica el proveedor.

Con respecto a la cristalinidad, la difractometría de rayos X (XRD) muestra que el principal compuesto cristalográfico es el óxido de aluminio-níquel, registrando una alta probabilidad para la familia de la alúmina debido a los picos detectados en el análisis, lo que concuerda con el material utilizado. Además, el grado cristalográfico del material fue bajo y por esto se obtuvieron picos pocos definidos como se muestra en la Figura 1. Esto permite deducir que el material analizado presenta formas amorfas y que predomina la gamma-alúmina marcado por los picos señalados en la figura a $38^{\circ}, 45^{\circ}$ y $69^{\circ}$.

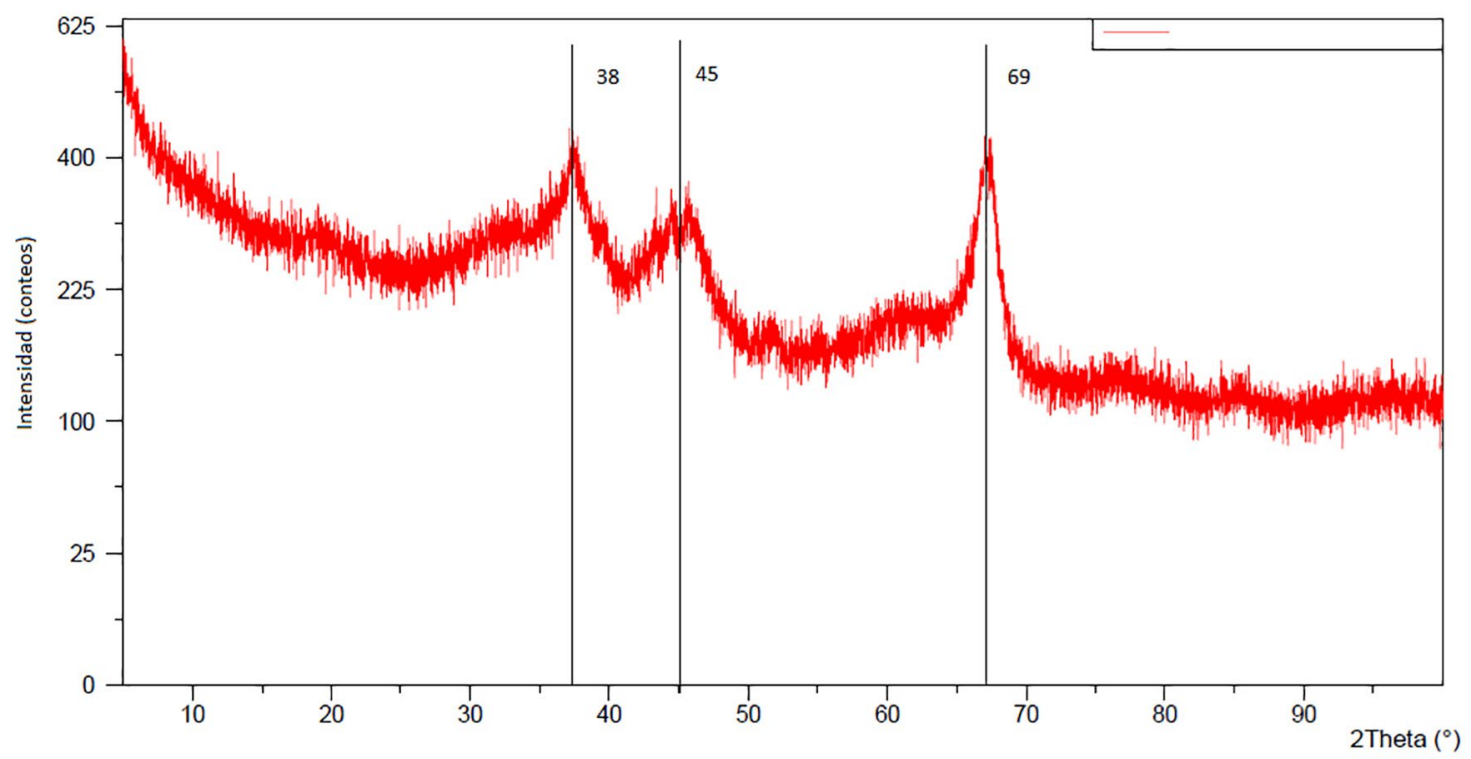

Figura 1. Difractograma de rayos X del catalizador utilizado.

El análisis de composición del catalizador impregnando, sin reducir ni calcinar, por medio de la técnica de absorción atómica, muestra que el porcentaje en masa de níquel fue de $1 \%$, sin embargo, este porcentaje cambia cuando se realiza el proceso de reducción, pues se elimina el oxígeno de la muestra. 
La microscopía electrónica (SEM) realizada al catalizador utilizado muestra irregularidad en el tamaño de la partícula (Figura 2), en donde se puede observar que el tamaño de las partículas que va desde los $50 \mu \mathrm{m}$ hasta los $150 \mu \mathrm{m}$.

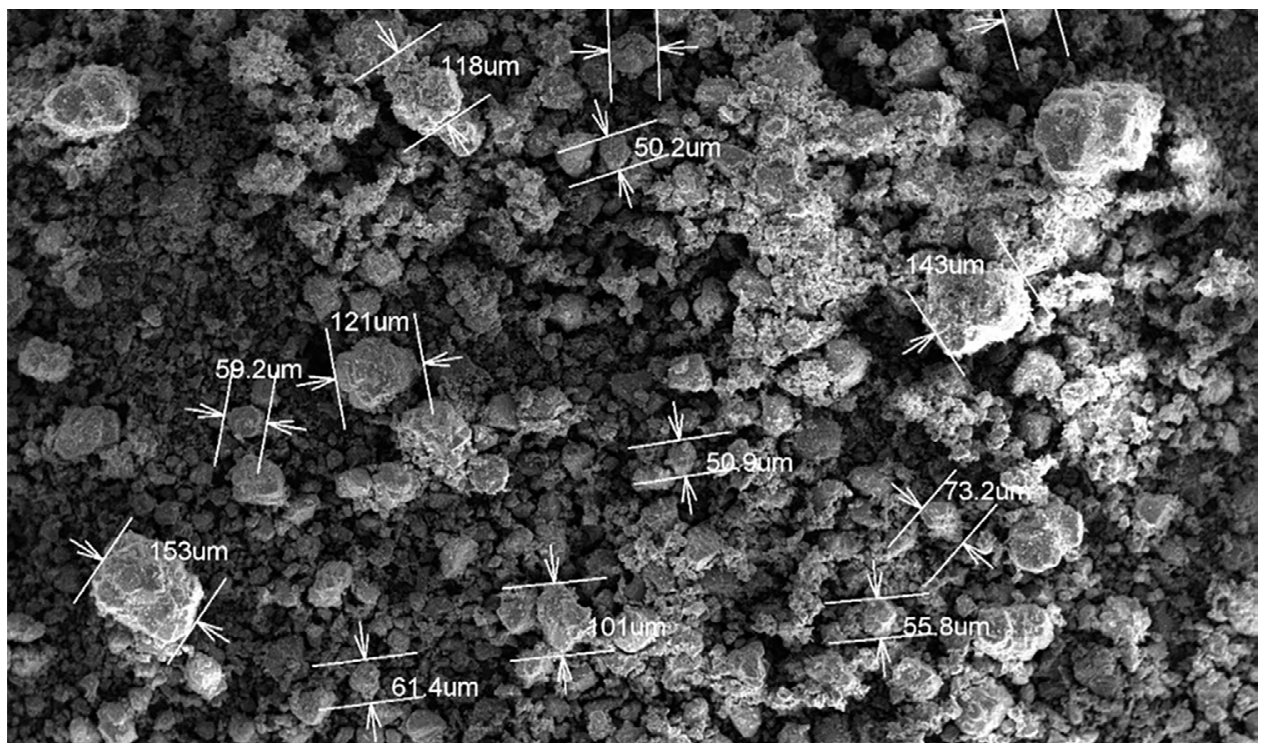

Figura 2. Micrografía SEM del catalizador utilizado.

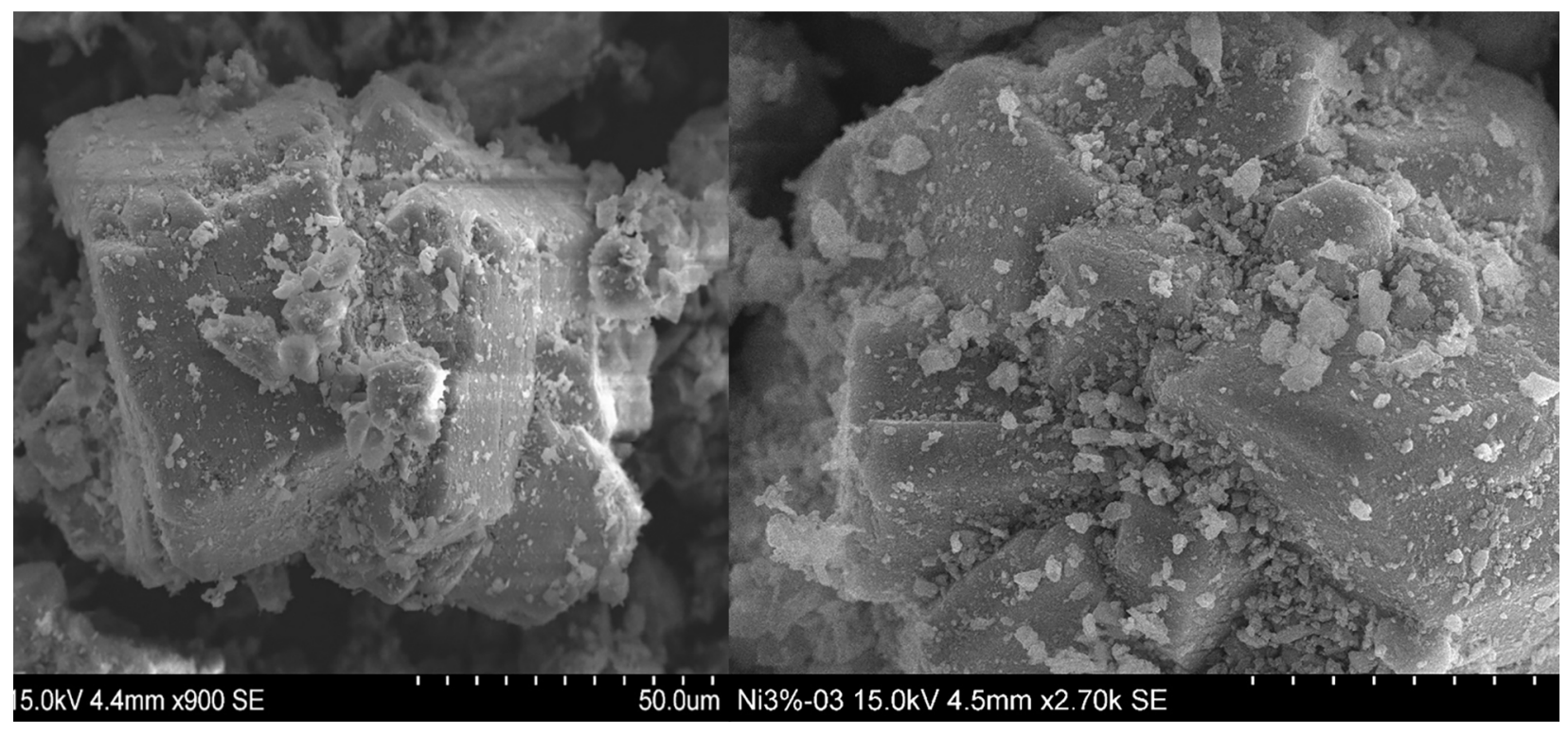

Figura 3. Micrografía SEM del catalizador utilizado.

Por medio del análisis de energía dispersiva de rayos X (EDX) realizado en el catalizador, se determina que el porcentaje de níquel de la muestra es similar al utilizado en el diseño experimental 
(3\%), y mayor al encontrado en el análisis de absorción atómica, ya que la muestra utilizada era catalizador reducido para este análisis. Con las micrografías como la que se muestra en la Figura 3, se evidencian zonas de mayor porcentaje de níquel, producto del proceso de impregnación del catalizador. Estas zonas son las partículas blancas pequeñas en donde los análisis reportan porcentajes de níquel del 4,8\%, sobre los gránulos más oscuros del soporte, donde se evidencian menores porcentajes de níquel, que variaron entre al $1,8 \%$ y $2,2 \%$.

\subsection{Producción de hidrógeno}

Se realizaron un total de ocho corridas experimentales, la variable respuesta que se escogió para el tratamiento estadístico fue la tasa de producción de hidrógeno por cantidad de catalizador, ya que el diseño experimental considera la variación de la cantidad de catalizador en las corridas. La elección del tiempo de reacción es una variable importante, ya que muchos estudios realizan las corridas hasta por 24 horas, pero por medio de pruebas preliminares se logró constatar que, entre las primeras horas de la reacción se llegó a una presión de equilibrio constante, lo cual permite delimitar un estado estable en el reactor; además permite disminuir la posibilidad del consumo de hidrógeno por reacciones secundarias como la hidrogenación y metanación, como sistemas paralelos a la reacción principal de craqueo de metanol, por lo que se definió el tiempo de reacción en dos horas a partir de que el sistema alcanzara la temperatura de trabajo.

En la Tabla 2 se tabulan los resultados para cada una de las corridas realizadas. Coronado et al. (2016), presentan un resumen de valores de producción de hidrógeno utilizando distintos sistemas de reacción, sustratos (glicerol, etanol, metanol) y condiciones de operación, con el mismo catalizador, en donde se observa que los valores obtenidos son similares a los que aparecen en la Tabla 2.

Tabla 2. Razón de producción de hidrógeno para los distintos niveles del diseño experimental

\begin{tabular}{ccccc}
\hline Corrida & Temperatura, $T /(K)$ & $\begin{array}{c}\text { Concentración de } \\
\text { Metanol, } C n /(\% \mathrm{v} / \mathrm{v})\end{array}$ & $\begin{array}{c}\text { Cantidad Catalizador, } \\
C C /(\% \mathrm{~m} / \mathrm{m})\end{array}$ & $\begin{array}{c}\text { Razón de producción } \\
\text { de } \mathrm{H}_{2}, \mathrm{G} / \\
\left(\mu \mathrm{mol} \mathrm{h}^{-1} / \mathrm{g} \text { cat }\right)\end{array}$ \\
\hline 1 & 473.15 & 2 & 2.5 & 1041 \\
2 & 473.15 & 2 & 5 & 1023 \\
3 & 473.15 & 6 & 2.5 & 539.2 \\
4 & 473.15 & 6 & 5 & 656.5 \\
5 & 498.15 & 2 & 2.5 & 2609 \\
6 & 498.15 & 2 & 5 & 2477 \\
7 & 498.15 & 6 & 2.5 & 2625 \\
8 & 498.15 & 6 & 5 & 2428 \\
\hline
\end{tabular}


La producción más alta que se obtuvo en este proyecto fue de $2.6 \mathrm{mmol} \mathrm{h}^{-1} / \mathrm{g}$ cat; Zhong Tang, Monroe, Junhang Dong, Nenoff y Weinkaut, 2009 reportan razones de producción de 0.70 mmol $\mathrm{h}^{-1} / \mathrm{g}$ cat, utilizando platino (3\%) soportado en alúmina a $498 \mathrm{~K}$; mientras que otros grupos de trabajo como el de Sakamoto, Kikuchi, Miyao, Yoshida y Naito (2010) reportan producciones de 78.4 $\mu \mathrm{mol} \mathrm{h} \mathrm{h}^{-1} / \mathrm{g}$ cat utilizando un catalizador de Pt-Ni (1:1) soportado en $\mathrm{TiO}_{2}$, a $357 \mathrm{~K}$ utilizando una alimentación de $10 \%$ de metanol.

En la Tabla 3 se presenta el análisis estadístico. Se utilizó un nivel de confianza del 95\% para la prueba realizada.

Tabla 3. Resumen de cálculos estadísticos para el diseño Taguchi L8

\begin{tabular}{ccccc}
\hline Fuente & $\begin{array}{c}\text { Grados de Libertad } \\
(G L)\end{array}$ & $\begin{array}{c}\text { Suma de Cuadros del } \\
\text { Modelo }(\text { Seq SS) }\end{array}$ & Factor F & Probabilidad \\
\hline Temperatura T/ $\left({ }^{\circ} \mathrm{C}\right)$ & 1 & 5915080 & 203.89 & 0.000 \\
$\begin{array}{c}\text { Concentración de Me- } \\
\text { tanol Cn/ } / \% \text { v/v) }\end{array}$ & 1 & 101926 & 3.521 & 0.134 \\
$\begin{array}{c}\text { Cantidad de catalizador } \\
\mathrm{CC} /(\% \mathrm{~m} / \mathrm{m})\end{array}$ & 1 & 6670 & 0.230 & 0.657 \\
Error Residual & 4 & 116042 & & \\
Total & 7 & 6139719 & & \\
\hline
\end{tabular}

Del análisis estadístico realizado se observa que la temperatura es la única variable significativa que afecta la producción de hidrógeno al pasar del nivel menor de trabajo (473.15 K) al valor mayor de trabajo (498.15 K), mientras que la concentración de metanol y la cantidad de catalizador no afectan la producción de hidrógeno dentro de las condiciones definidas en el diseño experimental.

Al realizar el análisis de los promedios del diseño Taguchi se observó que la temperatura fue la variable de mayor peso en la tasa de producción de hidrógeno, ya que una variación de $25 \mathrm{~K}$ provocó un cambio en la producción de hidrógeno mayor a $1500 \mu \mathrm{mol} \mathrm{h}^{-1} \mathrm{~g}_{\text {cat }}{ }^{-1}$; y en donde los valores de mayor producción se obtienen si se trabaja en la mayor temperatura (498.15 K).

El porcentaje de metanol es la segunda variable de importancia, ya que un cambio de un $4 \%$ v/v en la concentración del reactivo de interés solo modifica la concentración de hidrógeno producida en $225 \mu \mathrm{mol} \mathrm{h}{ }^{-1}$ gcat $^{-1}$. El nivel bajo (2\%) de metanol es el que consigue las mayores tasas de producción, lo que resulta ventajoso ya que a la hora de trabajar con disoluciones de metanol que son subproductos de otros procesos como la producción de etanol, fermentación de biomasas u otros bioprocesos se pueden esperar una variación natural del proceso y aun así, la producción 
promedio para el hidrógeno se podría mantener, permitiendo una flexibilidad ante las fluctuaciones de concentración de la materia prima que se puedan presentar.

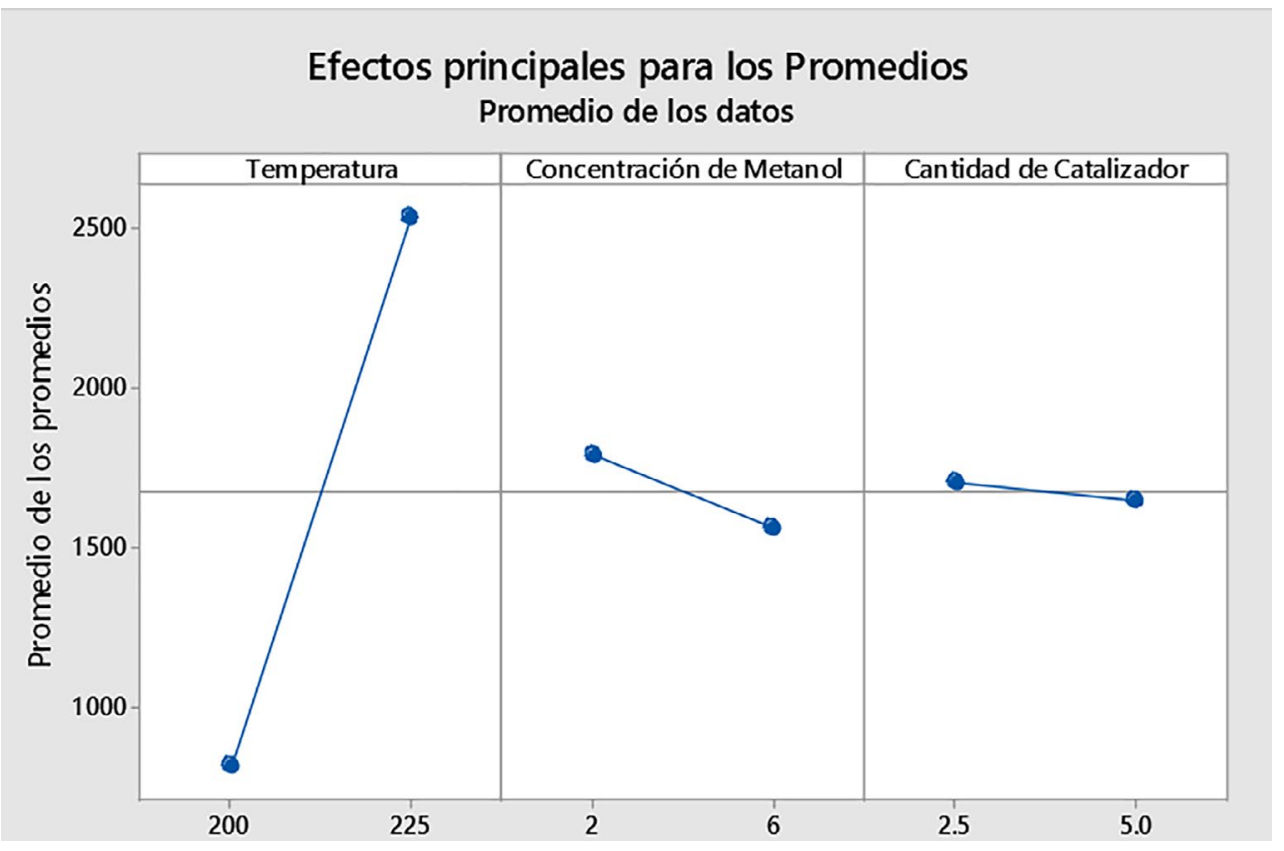

Figura 4. Valores promedio de producción de hidrógeno para las variables utilizadas en el diseño Taguchi L8.

Adicionalmente con respecto a estos resultados, es claro que, aunque se duplique la cantidad de catalizador el resultado es básicamente el mismo en la producción de hidrógeno; además que es mejor trabajar con la concentración más baja, lo que puede resultar atractivo desde un punto de vista económico. En la Figura 4 se puede ver las tendencias para las variables estudiadas.

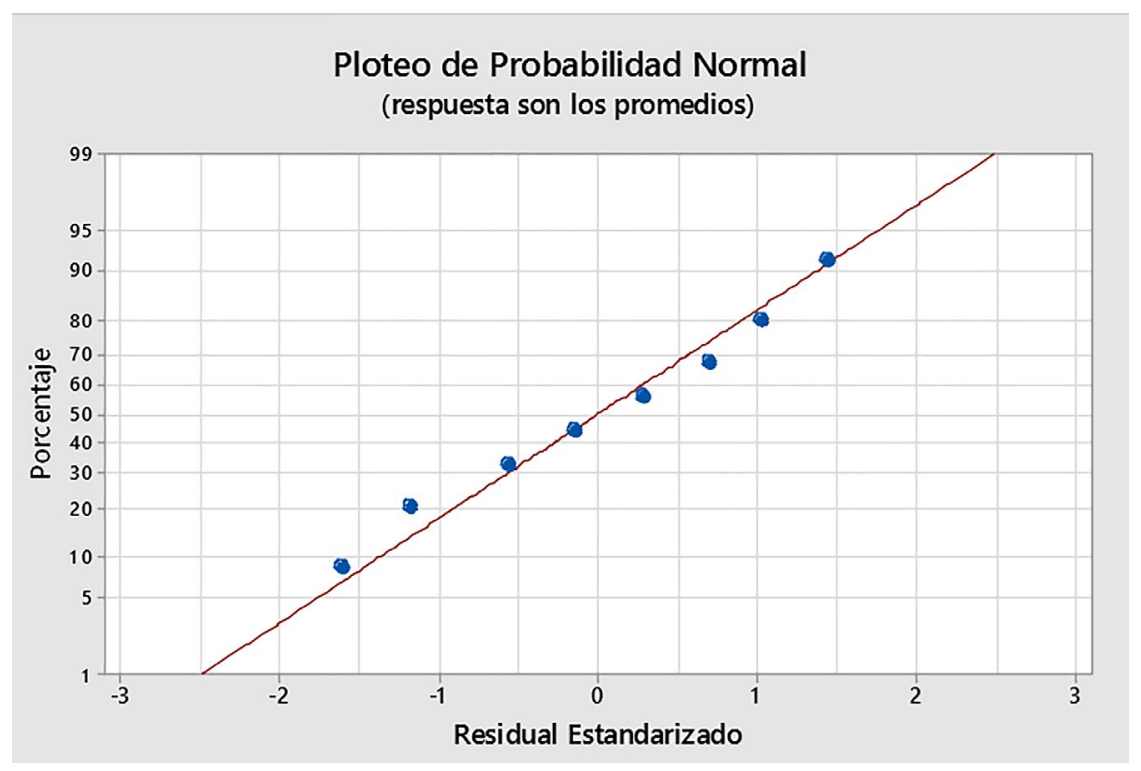

Figura 5. Curva de normalidad. 
Los resultados del diseño se validaron por medio de la gráfica de normalidad como se muestra en la Figura 5.

Asimismo, para confirmar el funcionamiento del catalizador y las condiciones de reacción, se realizó una corrida adicional en condiciones intermedias de $485.15 \mathrm{~K}\left(212{ }^{\circ} \mathrm{C}\right), 4 \%$ de metanol en disolución y $3.75 \mathrm{~g}$ de catalizador sin calcinación ni reducir. Como producto de esta corrida, la generación de hidrógeno fue de cero $(0) \mu \mathrm{mol} \mathrm{h}^{-1} \mathrm{gcat}^{-1}$, lo que confirma que el proceso de calcinación y reducción es necesario, que el óxido de níquel o la sal de níquel no catalizan en la reacción; y que bajo las condiciones de temperatura y reacción determinadas por la corrida en condiciones intermedias no se produce hidrógeno.

Con las tasas de producción de hidrógeno alcanzadas y bajo las condiciones de operación, se puede utilizar como un primer paso para valorizar productos secundarios de procesos agroindustriales, en este caso el metanol, que es un subproducto de la producción de etanol en la industria azucarera nacional. El metanol que se obtiene debe de ser tratado para su uso como combustible (y es medio de almacenamiento de energía más eficiente que el mismo hidrógeno); sin embargo, la integración de reformado en fase acuosa al proceso de producción de azúcar podría generar expectativas en otros mercados como es la producción de hidrógeno con una huella de carbono mínima, en comparación con las fuentes actuales de producción de hidrógeno comerciales.

\subsection{Desactivación del catalizador}

Se realizó un análisis de reutilización del catalizador, en el cual se evaluó el efecto en la tasa de producción de hidrógeno. Para esto se seleccionaron los valores de las variables de estudio como los niveles de la Corrida 6 de la Tabla 2; esto ya que se trabaja en los niveles altos de temperatura $(498.15 \mathrm{~K})$ y bajo de concentración de metanol $(2 \% \mathrm{v} / \mathrm{v})$, que fueron los que produjeron mayor cantidad de hidrógeno; y si bien es cierto las corridas 5 y 7 (Tabla 2) poseen una taza de producción de hidrógeno mayor cuando se utiliza un $2.5 \%$ de catalizador; al ser la variable con menor peso de variación en la producción de hidrógeno se decidió utilizar el nivel de 5\% de cantidad de catalizador por facilidad de preparar el sistema de reacción y para considerar la recuperación después de cada corrida.

Se determina repetir el experimento cuatro veces, ya que según estudios preliminares de desactivación esa cantidad de corridas permite obtener un panorama y tendencias de comportamiento del catalizador.

En la Figura 6 se observa que el proceso de desactivación del catalizador alcanza un 5,7\% de reducción en la tasa de producción de hidrógeno hasta el cuarto ciclo de utilización; no obstante, la diferencia entre los ciclos 1 a 3 es menor al $0,5 \%$. Si se considera la pérdida de catalizador por corrida realizada debido al lavado con el sistema de reacción, los porcentajes son menores al 1\%, excepto para el ciclo 3 , donde se perdió alrededor de un 5\% de masa de catalizador.

Hay que tomar en consideración que, aunque el uso del catalizador fue de 8 horas de acuerdo con el diseño experimental; este no fue un proceso continuo y más bien tomó 48 horas para realizar las mediciones considerando etapas de secado, limpieza y preparación del sistema de reacción, 
por lo que el tiempo de oxidación u otros procesos de desactivación para el catalizador es superior al tiempo de reacción estimado en el diseño experimental.

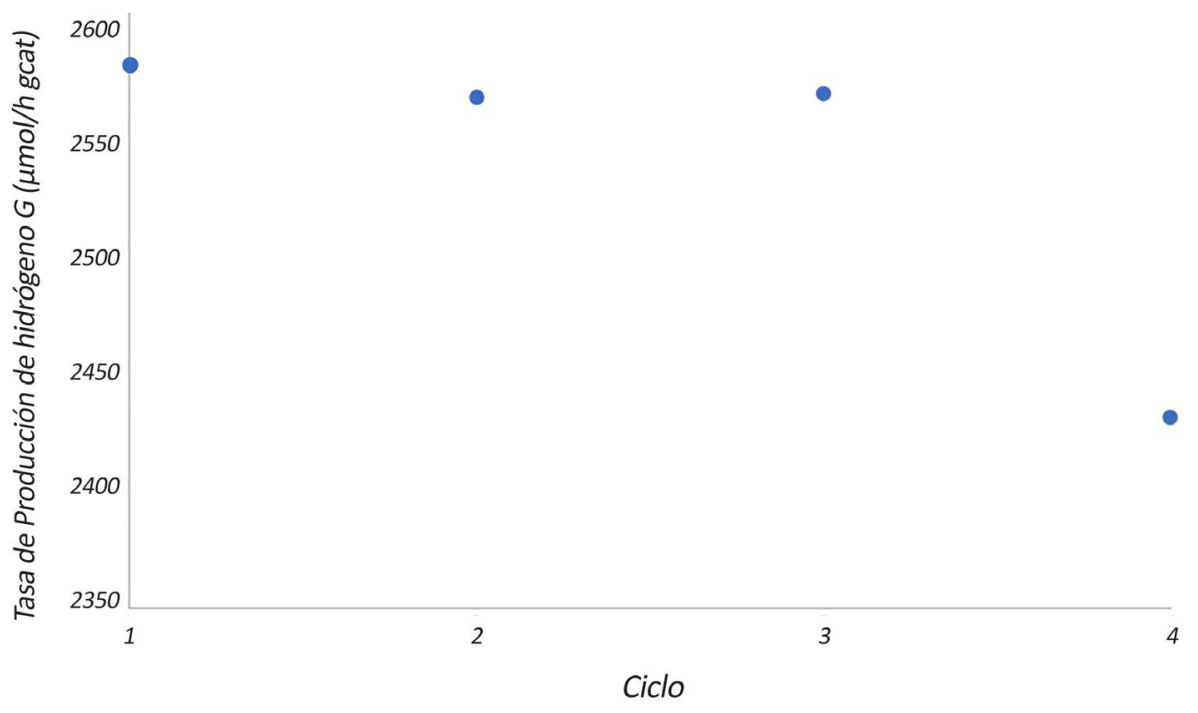

Figura 6. Proceso de desactivación del catalizador a base de Ni.

\section{CONCLUSIONES}

- Basados en los niveles utilizados en el diseño experimental, la condición de 498.15 K, $6 \%$ de metanol y $2.5 \mathrm{~g}$ de catalizador fue la que tuvo mejor desempeño para el catalizador de níquel soportado en alúmina con una tasa de producción de hidrógeno de $2625 \mu \mathrm{mol} \mathrm{h}^{-1} \mathrm{~g}_{\text {cat }}{ }^{-1}$.

- Se determinó que el área superficial de la alúmina comercial utilizada como soporte para el agente metálico del catalizador fue de $88 \mathrm{~m}^{2} / \mathrm{g}$ y concuerda dentro del rango aportado por el fabricante; sin embargo, es un valor bajo para la utilización como soporte en procesos de reacción.

- En las variables estudiadas por medio del análisis estadístico Taguchi L8, solo la temperatura es significativa y la condición que aumenta la taza de producción de hidrógeno es el valor de $498.15 \mathrm{~K}$.

- La cantidad de metanol en disolución y catalizador en suspensión no presentan diferencias significativas para la tasa de producción de hidrógeno, siendo la cantidad de catalizador la que presento menor diferencia entre los niveles trabajados.

\section{AGRADECIMIENTOS}

A la Red de Investigación y Desarrollo en Eficiencia Energética y Energías Renovables de Universidad de Costa Rica por el apoyo con el préstamo de equipos necesarios para el desarrollo del proyecto. 


\section{REFERENCIAS}

Cammack, R., Frey, M., y Robson, R. (2001). Hydrogen as a Fuel: Learning from Nature. CRC Press.

Chang, F.-W., Kuo, M.-S., Tsay, M.-T., y Hsieh, M.-C. (2003). Hydrogenation of CO2 over Nickel Catalysts on Rice husk Ash-alumina Prepared by Incipient Wetness Impregnation. Applied Catalysis A: General, 309-320.

Coronado, I., Stekrova, M., Reinikainen, M., Simell, P., Lefferts, L., y Lehtonen, J. (2016). A Review of Catalytic Aqueous-phase Reforming of Oxygenated Hydricarbons Derived from Biorefinery Water Fractions. International Journal of Hydrogen energy, 11003-11032.

Cruz, I. O., Ribeiro, N. F., Aranda, D. A., y Souza, M. M. (2008). Hydrogen Production by Aqueous-phase Reforming of Ethanol Over Nickel Catalysts Prepared from Hydrotalcite Precursors. Catalysis Communications, $2606-2611$.

Davda, R., Shabaker, J., Huber, G., Cortright, R., y Dumestic, J. (2005). A Review of Catalytic Issue and Process Conditions for Renewable Hydrogen and Alkenes by Aqueous-phase Reforming of Oxigenated Hydrocarbons Over Supported Metal Catalysts. Applied Catalysis B: Environmental, 171-186.

Incer, J. (2016). Efecto de la potencia y tiempo de irradiación de microondas utilizados en la preparación de fotocatalizadores sensibilizados de dióxido de titanio (TiO2) sobre sus rendimientos de producción de hidrógeno por separación de agua. San José: Universidad de Costa Rica.

Jose, S., y Shibu, T. (2015). Biomass and Biofuels, Advanced Biorefineries for Sustainable Production and Distribution. Boca Raton: CRC Press.

Miranda Morales, B. C. (2017). Effect of Preparation Method on Catalytic Activity of Ni/ y-Al2O3 Catalysts. Revista Ingeniería 27 (1): 21-38.

Munnik, P., de Jongh, P., y de Jong, K. (2015). Recent Developments in the Synthesis of Supported Catalysts. Chemical Reviews, 6687-6718.

Rase, H. F. (2016). Handbook of Commercial Catalysts: Heterogeneous Catalysts. Boca Raton: CRC Press.

Sakamoto, T., Kikuchi, H., Miyao, T., Yoshida, A., y Naito, S. (2010). Effect of Transition Metal Element Addition upon Liquid Phase Reforming of Methanol with Water over TiO2 Supported Pt Catalysts. Applied Catalysis A: General, 156-162.

Shabaker, J., Davda, R., Huber, G., Cortright, R., y Dumesic, J. (2003). Aqueous-phase reforming of methanol and ethylene glycol over alumina-supported platinum catalysts. Journal of Catalysis, 344-352.

Shan, Y. T. (2014). Water for Energy and Fuel Production. Boca Raton: CRC Press.

Stekrova, M., Rinta-Paavola, A., y Karinen, R. (2018). Hydrogen Production Via Aqueous-phase Reforming of Methanol over Nickel Modified Ce, Zr and La oxide Supports. Catalysis Today, 143-152.

Tungal, R., y Shende, R. (2013). Subcritical Aqueous Phase Reforming of Wastepaper for Biocrude and H2 Generation. Energy and Fuels, 3194-3203.

Valenzuela, M., Jones, C., y Agrawal, P. (2006). Batch Aqueous-Phase Reforming of Woody Biomass. Energy and Fuels, 1744-1752.

Zhong Tang, Monroe, J., Junhang Dong, Nenoff, T., y Weinkaut, D. (2009). Platinum-Loaded NaY Zeolita for Aqueous Phase Reforming of Methanol ans Ethanol to Hydrogen. Industrial Engineering Chemestry Research, 2728-2733. 